\title{
A class of equations with peakon and pulson solutions (with an Appendix by Harry Braden and John Byatt-Smith)
}

\author{
Darryl D. Holm*广and Andrew N.W. Hone ${ }^{\ddagger}$
}

June 4, 2018

To Francesco Calogero on the Occasion of his 70th Birthday

\begin{abstract}
We consider a family of integro-differential equations depending upon a parameter $b$ as well as a symmetric integral kernel $g(x)$. When $b=2$ and $g$ is the peakon kernel (i.e. $g(x)=\exp (-|x|)$ up to rescaling) the dispersionless Camassa-Holm equation results, while the Degasperis-Procesi equation is obtained from the peakon kernel with $b=3$. Although these two cases are integrable, generically the corresponding integro-PDE is non-integrable. However, for $b=2$ the family restricts to the pulson family of Fringer \& Holm, which is Hamiltonian and numerically displays elastic scattering of pulses. On the other hand, for arbitrary $b$ it is still possible to construct a nonlocal Hamiltonian structure provided that $g$ is the peakon kernel or one of its degenerations: we present a proof of this fact using an associated functional equation for the skew-symmetric antiderivative of $g$. The nonlocal bracket reduces to a non-canonical Poisson bracket for the peakon dynamical system, for any value of $b \neq 1$.
\end{abstract}

\section{Introduction}

Here we will consider a class of integro-partial differential equations (IPDEs) of the form

$$
m_{t}+u m_{x}+b u_{x} m=0, \quad u=g * m,
$$

where $g *$ denotes convolution with a symmetric integral kernel $g(x)$ defined on the real line $\mathbb{R}:$

$$
u(x)=g * m(x)=\int_{-\infty}^{\infty} g(x-y) m(y) d y .
$$

There are some distinguished special cases of this equation, the first being the dispersionless version of the integrable Camassa-Holm equation,

$$
u_{t}-u_{x x t}+3 u u_{x}=2 u_{x} u_{x x}+u u_{x x x}
$$

*Computer and Computational Science Division, Los Alamos National Laboratory, MS D413, Los Alamos, NM 87545. E-mail: dholm@lanl.gov

${ }^{\dagger}$ Mathematics Department, Imperial College London, SW7 2AZ, U.K. E-mail: d.holm@imperial.ac.uk

${ }^{\ddagger}$ Institute of Mathematics and Statistics, University of Kent, Canterbury CT2 7NF, U.K. E-mail: A.N.W.Hone@kent.ac.uk 
which is the $b=2$ case of (1.1) when the kernel is chosen to be the Green's function for the Helmholtz operator on the line:

$$
g(x)=\frac{1}{2} e^{-|x|}, \quad m=u-u_{x x} .
$$

With the inclusion of additional linear dispersion terms the equation (1.2) was derived as an approximation to the incompressible Euler equations, and found to be completely integrable with a Lax pair and associated bi-Hamiltonian structure [6, 7] (for another derivation, see [31]). In fact the Camassa-Holm equation fits into the framework of hereditary symmetries and recursion operators described earlier by Fokas and Fuchssteiner [23]. A remarkable discovery of [6, 7] was that in the dispersionless limit (1.2), the solitons of the CamassaHolm equation are peakons, given by a superposition of an arbitrary number of peaks,

$$
u(x, t)=\frac{1}{2} \sum_{j=1}^{N} p_{j}(t) e^{-\left|x-q_{j}(t)\right|},
$$

(See also [2, 10, 11].) The $N$-peakon solutions (1.4) are weak solutions with discontinuous first derivatives at the positions $q_{j}$ of the peaks; $q_{j}, p_{j}$ are canonical coordinates and momenta in an integrable finite-dimensional Hamiltonian system; the interpretation of weak solutions is discussed further in [12. The $N$-peakon interaction was obtained in [3, with the explicit peakon-antipeakon interaction being given in [4. The stability of peakons in the CamassaHolm equation was proved in [13, 14].

In a recent application of the method of asymptotic integrability to a many-parameter family of third order equations with dispersion, Degasperis and Procesi [17] found that only three equations passed the test up to third order in the asymptotic expansion, namely KdV, Camassa-Holm and one new equation. After rescaling and applying a Galilean transformation, the new equation may be written in dispersionless form as

$$
u_{t}-u_{x x t}+4 u u_{x}=3 u_{x} u_{x x}+u u_{x x x} .
$$

Henceforth we refer to (1.5) as the Degasperis-Procesi equation, and observe that it is the $b=3$ case of the equation (1.1) with $g(x)=e^{-|x|} / 2$. In a recent article [18] we proved the integrability of the Degasperis-Procesi equation by constructing a Lax pair, and derived two infinite sequences of conservation laws.
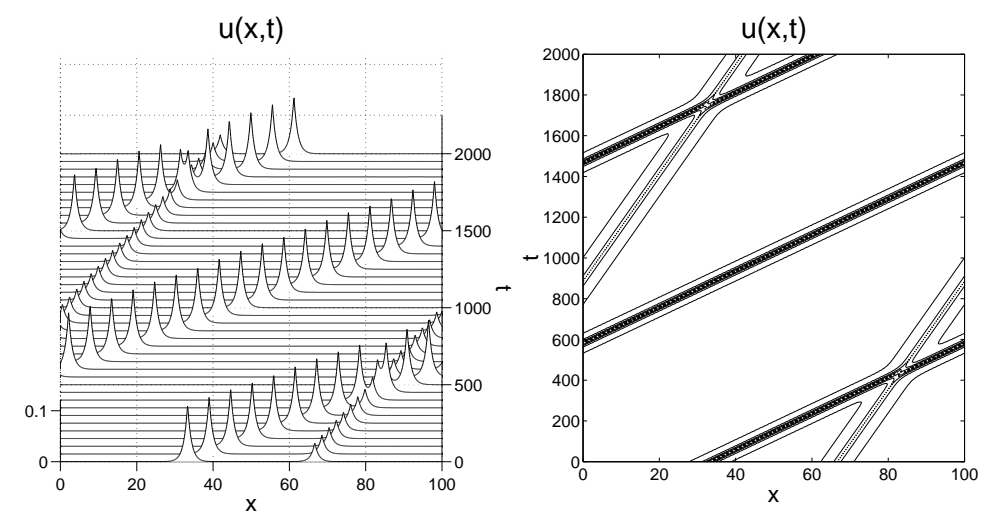

Figure 1: The elastic scattering of two peakons in the integrable Degasperis-Procesi equation on a periodic domain. This corresponds to $b=3$ and $g(x)=e^{-|x|} / 2$ in (1.1).

In order to better understand the common properties of the two integrable equations (1.2) and (1.5), we have found it convenient to consider the following one-parameter family 
of partial differential equations (PDEs):

$$
u_{t}-u_{x x t}+(b+1) u u_{x}=b u_{x} u_{x x}+u u_{x x x} .
$$

Since it arises from (1.1) when the peakon kernel (1.3) is chosen, we refer to (1.6) as the peakon $b$-family of PDEs. Remarkably, every member of this family admits multi-peakon solutions (1.4), with a non-canonical Hamiltonian structure for the dynamics of $q_{j}, p_{j}$ found in [19. The two-body dynamics is integrable for any $b$ (see Figure 1), and for all values of $b>1$ the peakons appear to be numerically stable and dominate the initial value problem 24. Furthermore, with the addition of linear dispersion $\left(u_{x}\right.$ and $u_{x x x}$ terms) it has been shown that not only the Camassa-Holm equation [20] but also the whole $b$-family (1.6) (apart from $b=-1$ ) belong to a class of asymptotically equivalent shallow water wave equations 21.

In [19] we presented a Lagrangian formulation for the $b$-family (1.6), with a Legendre transformation leading to the Hamiltonian structure

$$
m_{t}=\hat{B} \frac{\delta H}{\delta m}
$$

where for $b \neq 1$

$$
\hat{B}=-\left(b m \partial_{x}+m_{x}\right)\left(\partial_{x}-\partial_{x}^{3}\right)^{-1}\left(b m \partial_{x}+(b-1) m_{x}\right), \quad H=\frac{1}{b-1} \int m d x .
$$

(In the case $b=1$ it is necessary to take $H=\int m \log m d x$ instead.) The skew-symmetric operator $\hat{B}$ in (1.8) was first obtained in [18] for the case $b=3$, as a second Hamiltonian structure for the Degasperis-Procesi equation (1.5). However, a proof of the Jacobi identity for this operator was lacking until the work of one of us with Wang [29], where the trivector formalism of Olver [35] was applied to this problem. The first two Hamiltonian structures of (1.5) are

$$
B_{0}=-\partial_{x}\left(1-\partial_{x}^{2}\right)\left(4-\partial_{x}^{2}\right), \quad B_{1}=\left.\hat{B}\right|_{b=3}=-9 m^{2 / 3} \partial_{x} m^{1 / 3}\left(\partial_{x}-\partial_{x}^{3}\right)^{-1} m^{1 / 3} \partial_{x} m^{2 / 3},
$$

while for the Camassa-Holm equation (1.2) the bi-Hamiltonian structure arising from the Lax pair in [6, 7] is

$$
B_{0}=-\partial_{x}\left(1-\partial_{x}^{2}\right), \quad B_{1}=-\left(m \partial_{x}+\partial_{x} m\right)
$$

and applying the recursion operator $R=B_{1} B_{0}^{-1}$ to $B_{1}$ gives the nonlocal operator

$$
B_{2} \equiv B_{1} B_{0}^{-1} B_{1}=\left.\hat{B}\right|_{b=2} \text {. }
$$

Moreover, it is proved in [29] that $b=2,3$ are the only parameter values for which the operator $\hat{B}$ given by (1.8) is compatible with another operator with constant coefficients. The integrable cases $b=2,3$ can also be isolated by the perturbative symmetry approach 33], by the Wahlquist-Estabrook prolongation algebra method [29], or by using a reciprocal transformation and then applying Painlevé analysis [30].

In the special case of $b=2$ with an arbitrary symmetric kernel $g(x)$, the equation (1.1) has the Lie-Poisson structure

$$
m_{t}=\{m, \tilde{H}\}_{L P}:=-\left(m \partial_{x}+\partial_{x} m\right) \frac{\delta \tilde{H}}{\delta m}=-a d_{u}^{*} m,
$$


with the quadratic Hamiltonian

$$
\tilde{H}=\frac{1}{2} \int m g * m d x, \quad \frac{\delta \tilde{H}}{\delta m}=u .
$$

Equivalently the equation (1.10) corresponds to the Euler-Poincare equation for geodesic motion on the diffeomorphism group of the real line, assuming that $g$ (the co-metric) is the Green's function for a positive definite operator (see [22] for more details, and see [26] for an extension to higher dimensions). In the periodic case the Camassa-Holm equation is the equation for geodesics on the diffeomorphism group of the circle [34, leading to a proof of the corresponding least action principle [15, 16].

In the case $b=2$ given by (1.10) the equation admits special solutions in the form of a finite superposition of pulsons whose shape is determined by $g$, that is

$$
u=\sum_{j=1}^{N} p_{j}(t) g\left(x-q_{j}(t)\right), \quad m=\sum_{j=1}^{N} p_{j}(t) \delta\left(x-q_{j}(t)\right) .
$$

The finite-dimensional dynamical system for the motion of $N$ pulsons on the line is a canonical Hamiltonian system for geodesic motion on an $N$-dimensional manifold with coordinates $q_{j}, j=1, \ldots, N$ and co-metric $g\left(q_{j}-q_{k}\right)$, derived from the Hamiltonian

$$
\tilde{h}=\frac{1}{2} \sum_{j, k} p_{j} p_{k} g\left(q_{j}-q_{k}\right)
$$

The canonical Poisson bracket

$$
\left\{q_{j}, p_{k}\right\}=\delta_{j k} \quad\left\{q_{j}, q_{k}\right\}=0=\left\{p_{j}, p_{k}\right\}
$$

can be obtained by substituting the ansatz (1.11) for $m$ into the Lie-Poisson bracket

$$
\{m(x), m(y)\}_{L P}
$$

defined by the Hamiltonian operator $-\left(m \partial_{x}+\partial_{x} m\right)$, and similarly $\tilde{h}$ in (1.12) is derived from $\tilde{H}$ in (1.10) by substitution. Furthermore, although the equation (1.10) is generically non-integrable, numerical studies in [22] provide strong evidence that the pulson solutions (1.11) are stable and are produced by arbitrary smooth initial data, just as for the integrable case of peakons in the Camassa-Holm equation. We have proved non-integrability for a particular fifth order equation in this class [25], although numerically the pulsons appear to scatter elastically (see figure 2).

One might wonder why we have chosen to discuss this subject in a birthday volume in honour of Francesco Calogero. In fact Calogero has already studied the problem of finding Lax pairs for integrable finite-dimensional systems with geodesic Hamiltonians of the form (1.12), and obtained functional equations in terms of the function $g(x)$ and the components of the Lax matrix (see [8] and references). Here we are concerned with a slightly different problem. It turns out that the pulson ansatz (1.11) works equally well for the whole family of IPDEs (1.1), and leads to the dynamical system

$$
\frac{d q_{j}}{d t}=\sum_{k=1}^{N} p_{k} g\left(q_{j}-q_{k}\right), \quad \frac{d p_{j}}{d t}=-(b-1) \sum_{k=1}^{N} p_{j} p_{k} g^{\prime}\left(q_{j}-q_{k}\right) .
$$

Clearly this takes the canonical Hamiltonian form, with the Hamiltonian $\tilde{h}$ given by (1.12), only for the case $b=2$. However, in the particular case $b=3$ and $g(x)=e^{-|x|} / 2$ that 

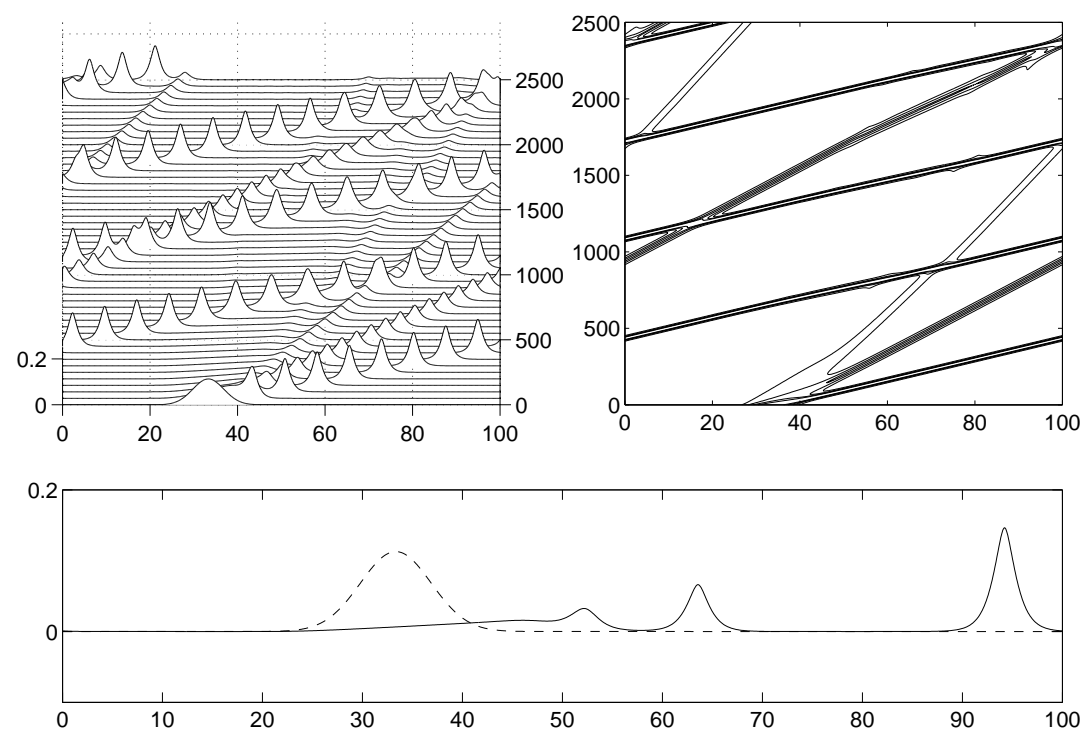

Figure 2: Pulson solutions (1.11) of equation (1.10) with $g(x)=2 e^{-|x|}-e^{-2|x|}$. emerge from a Gaussian of unit area and width $\sigma=5$ centered about $x=33$ on a periodic domain of length $L=100$. The fastest pulson crosses the domain four times and collides elastically with the slower ones.

corresponds to the integrable Degasperis-Procesi equation, we have derived a matrix Lax pair for the system (1.14), and therefore in this case the system must be integrable with respect to some Poisson structure. Requiring the existence of a certain type of Poisson structure for the IPDEs (1.1) leads us to the functional equation

$$
G^{\prime}(\alpha)(G(\beta)+G(\gamma))+G^{\prime}(\beta)(G(\gamma)+G(\alpha))+G^{\prime}(\gamma)(G(\alpha)+G(\beta))=0
$$

when $\alpha+\beta+\gamma=0$, for the antisymmetric kernel

$$
G(x)=\int_{0}^{x} g(t) d t
$$

Apart from some limiting cases, the general odd solution of (1.15) turns out to be

$$
G(x)=A \operatorname{sgn}(x)\left(1-e^{-B|x|}\right), \quad \text { whence } \quad g(x)=A B e^{-B|x|} \quad(A, B \text { constant }) .
$$

(The derivation of the general solution of (1.15) is presented in an Appendix provided by H.W. Braden and J.G. Byatt-Smith.) Thus the peakon $b$-family (1.6) is picked out of the class (1.1) by the functional equation (1.15). Moreover, for any $b$ the corresponding Hamiltonian operator, given by (1.8), reduces to a Poisson bracket for the $N$-peakon dynamical system, first presented without proof in [19]. In the next section we derive the functional equation from the Jacobi identity for the operator (1.8), and then proceed to show how this leads to a suitable bracket for the peakons.

Historical Note. This paper is dedicated to our friend, Francesco Calogero. This work parallels some of his earlier considerations on geodesic flows and the determination of integrability for dynamical systems by using functional equations. Calogero's fundamental works have been and remain incredibly influential and important to the field of integrable systems. Francesco himself is still developing the mathematics associated with this area. (Just look over the $200+$ papers on MathSciNet that he has written!) His contributions will surely influence the next generation of people working in this area, as they have with the current generation. Of Francesco's work, the references that are most directly relevant to the topics discussed in the present paper are [8, 9]. 


\section{Derivation of the functional equation}

Let us start from any equation (1.1) in the pulson $b$-family. The key is to introduce, for any kernel $g$, the operator

$$
\hat{B}=-\left(b m \partial_{x}+m_{x}\right) \hat{G}\left(b m \partial_{x}+(b-1) m_{x}\right),
$$

where the operator $\hat{G}$ acts according to

$$
\hat{G} f(x)=G * f=\int_{-\infty}^{\infty} G(x-y) f(y) d y,
$$

with $G$ being the antisymmetric kernel (1.16). Thus $\hat{B}$ is skew-symmetric and for $b \neq 1$ satisfies

$$
m_{t}=\hat{B} \frac{\delta H}{\delta m}, \quad H=\frac{1}{(b-1)} \int_{-\infty}^{\infty} m d x .
$$

Now we can calculate the bracket defined by the operator $\hat{B}$, namely

$$
\{F, G\}=\int_{-\infty}^{\infty} \frac{\delta F}{\delta m} \hat{B} \frac{\delta G}{\delta m} d x,
$$

which gives

$$
\begin{gathered}
\{m(x), m(y)\}=\left(G(x-y) m_{x}(x) m_{x}(y)+b G^{\prime}(x-y)\left(m(x) m_{x}(y)-m(y) m_{x}(x)\right)\right. \\
\left.-b^{2} G^{\prime \prime}(x-y) m(x) m(y)\right) .
\end{gathered}
$$

Although (2.2) is antisymmetric (since $G$ is odd), it only defines a Poisson bracket if the Jacobi identity is satisfied, i.e.

$$
\{\{m(x), m(y)\}, m(z)\}+\text { cyclic }=0 .
$$

To calculate (2.3), we evaluate

$$
\begin{gathered}
\frac{\delta}{\delta m(s)}\{m(x), m(y)\}=\left(G(x-y)\left[\delta^{\prime}(x-s) m_{x}(y)+\delta^{\prime}(y-s) m_{x}(x)\right]\right. \\
+b G^{\prime}(x-y)\left[\delta(x-s) m_{x}(y)-\delta(y-s) m_{x}(x)+\delta^{\prime}(y-s) m(x)-\delta^{\prime}(x-s) m(y)\right] \\
\left.-b^{2} G^{\prime \prime}(x-y)[\delta(x-s) m(y)+\delta(y-s) m(x)]\right),
\end{gathered}
$$

and then substitute into

$$
\{\{m(x), m(y)\}, m(z)\}=\int_{-\infty}^{\infty} \frac{\delta}{\delta m(s)}\{m(x), m(y)\} \hat{B}(s) \delta(z-s) d s
$$

(where $\hat{B}(s)$ indicates the operator acting in the independent variable $s$ ).

In the Jacobi identity (2.3) many terms cancel to leave only terms cubic in $m$ and $m_{x}$, whose coefficients must each vanish if the identity holds for arbitrary $m$. This leads to functional equations for the odd function $G$, but remarkably (apart from overall prefactors) these coefficients are independent of the parameter $b$. Thus, setting

$$
\alpha=x-y, \quad \beta=y-z, \quad \gamma=z-x, \quad \text { whence } \quad \alpha+\beta+\gamma=0,
$$


we find the coefficient of $m_{x}(x) m_{x}(y) m_{x}(z)$ yields the functional equation (1.15). The coefficient of $m(x) m_{x}(y) m_{x}(z)$ gives

$$
G^{\prime}(\beta)\left(G^{\prime}(\alpha)-G^{\prime}(\gamma)\right)-G^{\prime \prime}(\gamma)(G(\alpha)+G(\beta))+G^{\prime \prime}(\alpha)(G(\beta)+G(\gamma))=0,
$$

and there are two other identities like (2.4) obtained by cyclic permutations of $x, y, z$ which induce cyclic permutations of $\alpha, \beta, \gamma$ (with the three identities summing to zero). Similarly the coefficient of $m_{x}(x) m(y) m(z)$ yields

$$
G^{\prime \prime \prime}(\beta)(G(\alpha)+G(\gamma))+G^{\prime \prime}(\gamma)\left(G^{\prime}(\alpha)-G^{\prime}(\beta)\right)-G^{\prime \prime}(\alpha)\left(G^{\prime}(\beta)-G^{\prime}(\gamma)\right)=0
$$

and there are two other identities obtained by cyclic permutation of $\alpha, \beta, \gamma$ in (2.5). Finally the coefficient of $m(x) m(y) m(z)$ requires

$$
G^{\prime \prime \prime}(\alpha)\left(G^{\prime}(\beta)-G^{\prime}(\gamma)\right)+G^{\prime \prime \prime}(\beta)\left(G^{\prime}(\gamma)-G^{\prime}(\alpha)\right)+G^{\prime \prime \prime}(\gamma)\left(G^{\prime}(\alpha)-G^{\prime}(\beta)\right)=0
$$

There are several things to observe about these functional equations. Firstly, (2.4) and (2.5) are direct consequences of (1.15), obtained by differentiating. The final functional equation (2.6) is also a consequence of the rest. Hence the Jacobi identity is satisfied by the operator $\hat{B}$ if and only if the antisymmetric kernel $G$ satisfies the functional equation (1.15). We can prove the following:

Proposition. Suppose that $G(x)$ is an odd solution of the functional equation (1.15) with continuous second derivative $G^{\prime \prime}(x)$ for all $x \in \mathbb{R} \backslash\{0\}$, and suppose $\lim _{x \rightarrow 0 \pm} G^{\prime \prime}(x)$ is finite. Then either $G(x)=A x$ or $G(x)=A \operatorname{sgn}(x)\left(1-e^{-B|x|}\right)$ for some constants $A, B$.

Sketch of proof. If $G$ is odd and continous second differentiable then from the limit $\alpha \rightarrow 0$ in (1.15) we see that $G(0)=0$, while differentiating (1.15) with respect to $\alpha$ yields (2.4). Taking the limit $\alpha \rightarrow 0$ in (2.4) with $\beta$ fixed gives the differential equation

$$
G^{\prime \prime}(\beta) G(\beta)-G^{\prime}(\beta)^{2}+G^{\prime}(0) G^{\prime}(\beta)=0,
$$

which integrates to

$$
G^{\prime}(\beta)=G^{\prime}(0)+K_{ \pm} G(\beta)
$$

where the integration constants $K_{ \pm}$can be different for $\beta>0$ and $\beta<0$. This is easily integrated to give the solution for $G$.

Remarks. A more detailed derivation of the general solution (not necessarily odd), with weaker assumptions, appears as an Appendix. Here we should observe that if the parameter $B \rightarrow \infty$ with $A$ fixed then the distributional solution

$$
G(x)=A \operatorname{sgn}(x), \quad g(x)=2 A \delta(x)
$$

results, in which case for $A=1$ (1.1) becomes the Riemann shock equation

$$
m_{t}+2(b+1) m m_{x}=0
$$

while when $B \rightarrow 0$ with $A \sim 1 / B$ the linear solution $G(x)=A x$ arises. The latter gives only the trivial linear PDE

$$
m_{t}+K m_{x}=0, \quad K=A \int m(y) d y
$$




\section{$3 \quad$ Restricting the bracket to the pulsons}

To calculate the corresponding bracket for the restriction to the finite-dimensional pulson submanifold, we substitute $m=\sum p_{j} \delta\left(x-q_{j}\right)$ into both sides of (2.2), which gives

$$
\begin{gathered}
\sum_{j, k}\left(\left\{p_{j}, p_{k}\right\} \delta\left(x-q_{j}\right) \delta\left(y-q_{k}\right)-\left\{p_{j}, q_{k}\right\} p_{k} \delta\left(x-q_{j}\right) \delta^{\prime}\left(y-q_{k}\right)\right. \\
\left.-\left\{p_{j}, q_{k}\right\} p_{j} \delta^{\prime}\left(x-q_{j}\right) \delta\left(y-q_{k}\right)+\left\{q_{j}, q_{k}\right\} p_{j} p_{k} \delta^{\prime}\left(x-q_{j}\right) \delta^{\prime}\left(y-q_{k}\right)\right)= \\
\sum_{j, k} p_{j} p_{k}\left(G(x-y) \delta^{\prime}\left(x-q_{j}\right) \delta^{\prime}\left(y-q_{k}\right)+b G^{\prime}(x-y)\left(\delta\left(x-q_{j}\right) \delta^{\prime}\left(y-q_{k}\right)-\delta^{\prime}\left(x-q_{j}\right) \delta\left(y-q_{k}\right)\right)\right. \\
\left.-b^{2} G^{\prime \prime}(x-y) \delta\left(x-q_{j}\right) \delta\left(y-q_{k}\right)\right) .
\end{gathered}
$$

We fix $\epsilon$ with $0<\epsilon<\min _{j \neq k}\left|q_{j}-q_{k}\right|$ and then introduce the indicator functions

$$
\begin{aligned}
I_{j}(x) & =1, \quad x \in\left[q_{j}-\epsilon, q_{j}+\epsilon\right] \\
& =0, \quad \text { otherwise }
\end{aligned}
$$

and

$$
J_{j}(x)=\left(x-q_{j}\right) I_{j}(x) .
$$

Integrating the Poisson bracket relation against suitable products of indicator functions centred around $x=q_{j}, y=q_{k}$, such as $I_{j}(x) I_{k}(y)$ and $I_{j}(x) J_{k}(y)$ etc. we find

$$
\begin{gathered}
\left\{p_{j}, p_{k}\right\}=-(b-1)^{2} G^{\prime \prime}\left(q_{j}-q_{k}\right) p_{j} p_{k}, \quad\left\{q_{j}, p_{k}\right\}=(b-1) p_{k} G^{\prime}\left(q_{j}-q_{k}\right), \\
\left\{q_{j}, q_{k}\right\}=G\left(q_{j}-q_{k}\right) .
\end{gathered}
$$

The brackets (3.1) generate the pulson $b$-equations by taking the restriction of $H$ to the finite submanifold i.e.

$$
H=\frac{1}{(b-1)} \int_{-\infty}^{\infty} \sum_{k} p_{k} \delta\left(x-q_{k}\right) d x=\frac{1}{(b-1)} \sum_{k} p_{k}
$$

Thus we find that the pulson dynamical system (1.14) becomes

$$
\frac{d p_{j}}{d t}=\left\{p_{j}, H\right\}, \quad \frac{d q_{j}}{d t}=\left\{q_{j}, H\right\}
$$

(with $G^{\prime}=g$ ). But (3.1) are only Poisson brackets if the Jacobi identity is satisfied. For the Jacobi identity of the finite-dimensional brackets we require both

$$
\left\{\left\{q_{j}, q_{k}\right\}, p_{l}\right\}+\text { cyclic }=0=\left\{\left\{p_{j}, p_{k}\right\}, q_{l}\right\}+\text { cyclic } .
$$

for all $j, k, l$, and similarly

$$
\left\{\left\{q_{j}, q_{k}\right\}, q_{l}\right\}+\text { cyclic }=0=\left\{\left\{p_{j}, p_{k}\right\}, p_{l}\right\}+\text { cyclic. }
$$

The necessary conditions resulting from (3.2) and 3.3) are the functional equations (1.15), (2.4), (2.5), (2.6) and their cyclic permutations. Therefore if $G$ satisfies the functional equation (1.15) then this guarantees that (3.1) is a finite-dimensional Poisson bracket. 


\section{Conclusions}

The functional equation (1.15) provides an elegant way to pick out the peakon equations (1.6) from the whole class of IPDEs (1.1). However, it is interesting that the compacton type solution $G(x)=x(1-|x| / 2) H(1-x) H(1+x)$ does not satisfy the equation. The compactons are solitons with compact support which appear in the Hunter-Saxton [36] or Vakhnenko equations [37, 38. In that case $G$ is formally a Green's function for $\partial_{x}^{-3}$, and the operator (2.1) was formally identified as satisfying the Jacobi identity in 29]. However, in [29] we noted that the Vakhnenko equation is the short wave limit of the Degasperis-Procesi equation, and so there may be problems with defining the boundary conditions correctly.

Acknowledgements. We are grateful to Martin Staley for providing the Figures. Both authors would like to thank Toni Degasperis for introducing us to the Degasperis-Procesi equation. We are also grateful for the hospitality of the Isaac Newton Institute, where we first began working on $b=3$ in 2001. AH thanks Harry Braden and John Byatt-Smith for providing the general solution of the functional equation, and is grateful to Andrey Leznov for suggesting the simplest approach when $G$ is odd. DH and $\mathrm{AH}$ also thank Simonetta Abenda and Tamara Grava for inviting us to the meeting Analytic and Geometric theory of the Camassa-Holm equation and Integrable Systems in Bologna, in September 2004, where we decided to finish writing up these results. DH thanks AH for taking the lead in finishing our project and finally transforming it from a long string of email exchanges into a finished product. We are both grateful to Francesco Calogero for many kindnesses and inspiring discussions over the years of our association together.

\section{Appendix: Solution of the Functional Equation by H.W. Braden and J.G. Byatt-Smith}

Here we shall solve the functional equation

$$
G^{\prime}(x)[G(y)+G(z)]+G^{\prime}(y)[G(z)+G(x)]+G^{\prime}(z)[G(x)+G(y)]=0
$$

where $x+y+z=0$ and $G(x)$ is differentiable with continuous first derivative. We prove the following theorem.

Theorem 4.1. Suppose $G(x)$ is a solution of equation 4.1) that is differentiable with continuous first derivative. If $G(x)$ possesses (possibly different) power series expansions in the intervals $(-\epsilon, 0)$ and $(0, \epsilon)$ (for $\epsilon>0)$ then, up to the invariance of the functional equation

$$
G(x) \rightarrow a G(A x)
$$

$G(x)$ is one of

$$
\text { (i) } \quad G(x)=\frac{1}{c}\left[e^{A x}+e^{-A x}+1\right],
$$

which for $A=0$ gives the constant solution.

$$
G(x)=\sin (x) \sin \left(x-\frac{\pi}{3}\right),
$$

which has the scaling limit $G(x)=\alpha x$.

$$
G(x)=\operatorname{sgn}(x)\left[1-e^{-|x|}\right] .
$$

We begin with some preliminary observations. 
1. Equation (4.1) is satisfied for $G$ a constant. Henceforth we assume that $G$ does not vanish identically.

2. $G(0) G^{\prime}(0)=0$. This is seen by setting $0=x=y=z$ in (4.1).

3. Suppose that both $G(0)=G^{\prime}(0)=0$. Then with $x=0$ and $z=-y$ we find that

$$
G^{\prime}(y) G(-y)+G^{\prime}(-y) G(y)=0
$$

Thus $G(-y) / G(y)=\lambda$, a constant. Then $\lambda= \pm 1$, and therefore $G$ is either even or odd.

4. With $x=y$ we obtain

$$
G(-2 x) G^{\prime}(x)+G^{\prime}(x) G(x)+G^{\prime}(-2 x) G(x)=0,
$$

and so

$$
\frac{d}{d x}\left[\frac{G(-2 x)}{G^{2}(x)}+\frac{2}{G(x)}\right]=0 .
$$

Therefore, on an interval $I$ for which $G(x) \neq 0$ we have

$$
G(-2 x)+2 G(x)=c_{I} G^{2}(x)
$$

It is possible for the constant $c_{I}$ to vary from interval to interval. This means that the function can have different power series expansions in different intervals. There are solutions possessing different power series on different intervals. For example (4.5) satisfies (4.7) with $c=1$ for $x>0$ and $c=-1$ for $x<0$. (We will see how this arises below.)

Thus solutions of equation (4.7) contain those of (4.1).

5. From (4.1) we deduce that if $G^{\prime}$ is differentiable at the point $y$ and $G(y)+G(-x-y) \neq 0$, then $G^{\prime}$ is also differentiable at the point $x$. Thus if $G^{\prime}$ is differentiable for $0<\left|x_{0}-y\right|<$ $\epsilon$, but not at $x_{0}$ we have

$$
G(y)+G\left(-x_{0}-y\right)=0 .
$$

Using the continuity of $G$ we have

$$
G\left(x_{0}\right)+G\left(-2 x_{0}\right)=0 .
$$

We deduce from (4.6) that at such a point

$$
G\left(x_{0}\right) G^{\prime}\left(-2 x_{0}\right)=0 .
$$

6. We record

$$
\begin{aligned}
-G^{\prime}(-2 x) & =c_{I} G(x) G^{\prime}(x)-G^{\prime}(x) \\
2 G^{\prime \prime}(-2 x) & =c_{I}\left[G^{\prime}(x)^{2}+G(x) G^{\prime \prime}(x)\right]-G^{\prime \prime}(x) \\
-4 G^{\prime \prime \prime}(-2 x) & =c_{I}\left[3 G^{\prime}(x) G^{\prime \prime}(x)+G(x) G^{\prime \prime \prime}(x)\right]-G^{\prime \prime \prime}(x) \\
8 G^{(i v)}(-2 x) & =c_{I}\left[4 G^{\prime}(x) G^{\prime \prime \prime}(x)+3 G^{\prime \prime}(x)^{2}+G(x) G^{(i v)}(x)\right]-G^{(i v)}(x)
\end{aligned}
$$




\section{Proof of the Theorem}

The strategy of our proof is the following. We have observed that the solutions of equation (4.7) contain those of (4.1). We will first find the solutions of equation (4.7) satisfying the conditions of the theorem, and then verify that these in fact solve (4.1).

Observe from (4.7) that if $G(x)$ is defined in some neighbourhood of $x=0$ it may be extended to the whole of the real line. We will assume that $G(x)$ has a power series expansion for $0<|x|<\epsilon$, possibly different for $x$ positive and negative. To distinguish between the possibly different power series for $G$ set

$$
\begin{array}{ll}
g(x)=G(x), & \text { if } \quad x>0, \\
f(x)=G(-x), & \text { if } \quad x<0 .
\end{array}
$$

Then from (4.7)

$$
\begin{aligned}
& f(2 x)=c_{+} g(x)^{2}-2 g(x), \\
& g(2 x)=c_{-} f(x)^{2}-2 f(x) .
\end{aligned}
$$

In particular, this means that

$$
f(4 x)=c_{+}\left[c_{-} f(x)^{2}-2 f(x)\right]^{2}-2\left[c_{-} f(x)^{2}-2 f(x)\right] .
$$

Our assumptions on the continuity of $G$ and $G^{\prime}$ mean that $f(0)=g(0)$ and $f^{\prime}(0)=g^{\prime}(0)$. We also note from (4.7) that

$$
3 f(0)=c_{-} f(0)^{2}, \quad 3 g(0)=c_{+} g(0)^{2} .
$$

Now we two distinct cases, as follows:

Case (1) If $f(0)=g(0) \neq 0$, then from (4.13) we deduce that $c_{-}=3 / f(0)=3 / g(0)=c_{+}$. Therefore $f(x)$ and $g(x)$ satisfy the same recursion with the same initial conditions, whence $f(x)=g(x)$. Together with say (4.10) this yields a recursion for the power series coefficients of the function (see for example (4.8) ) which may be solved to give

$$
G(x)=\frac{1}{c}\left[e^{A x}+e^{-A x}+1\right] .
$$

With $A=0$ this gives $f(x)=3 / c$, the constant solution noted earlier.

Case (2) If $f(0)=g(0)=0$ then there are two possibilities:

(a) If $f^{\prime}(0)=g^{\prime}(0)=0$ then from our earlier observation we have that $f(x)= \pm g(x)$. Substituting the power series $f(x)=c_{2} x^{2}+\ldots$ into (4.10) and employing this observation shows $f(x)=0$, and consequently

$$
G(x)=0 .
$$

(b) Thus we may suppose $f^{\prime}(0)=g^{\prime}(0) \neq 0$. Now from (4.8) we find that in this case

$$
\begin{aligned}
& 3 f^{\prime \prime}(0)=\left(2 c_{+}-c_{-}\right) f^{\prime}(0)^{2}, \\
& 3 g^{\prime \prime}(0)=\left(2 c_{-}-c_{+}\right) g^{\prime}(0)^{2} .
\end{aligned}
$$

1. If the second derivatives agree then $c_{+}=c_{-}$and again $f(x)=g(x)$. Solving the recursion yields

$$
G(x)=\sin (x) \sin \left(x-\frac{\pi}{3}\right)=-\frac{\sqrt{3}}{2} x+\frac{x^{2}}{2}+\frac{x^{3}}{3}-\frac{x^{4}}{6}+\ldots
$$

This satisfies $G(-2 x)+2 G(x)=4 G^{2}(x)$; that is $c=4$. A scaling limit of this solution is

$$
G(x)=\alpha x
$$

which corresponds to $c=0$. (This is obtained by $G(x) \rightarrow a G(x)$, under which $c \rightarrow \frac{c}{a}$.) 
2. The final possibility corresponds to $f$ and $g$ having different second derivatives at $x=0$. This corresponds to $G^{\prime}$ not being differentiable at this point. Our earlier observation then yields $G(y)+G(-y)=0$. That is $G$ is odd, whence $f(x)=-g(x)$ and consequently $c_{+}=-c_{-}$. Solving the recursion yields

$$
G(x)=\operatorname{sgn}(x)\left[1-e^{-|x|}\right] .
$$

At this stage we have established the Theorem.

Remark. In [5] an alternative method is described for investigating functional equations like (4.1). It is instructive to compare this with the approach above. Upon setting $y=x-t$ and $z=t-2 x$ we expand (4.1) in $t$ : this leads to an infinite set of differential equations that $G$ must satisfy. The term independent of $t$ yields (4.6) and the equation from the order $t$ term is satisfied identically as a consequence of (4.7). If we substitute (4.7), which involves the arbitrary constant $c$, into the order $t^{2}$ and $t^{3}$ equations resulting from the expansion we can eliminate the constant $c$ between them. This yields the following simple result

$$
0=g(x) g^{\prime \prime}(x)\left[g^{(i v)} g^{\prime}(x)-g^{\prime \prime}(x) g^{\prime \prime \prime}(x)\right]
$$

from which we see that either $g$ vanishes, is a linear function, or satisfies the fourth order differential equation given by the last term. The general solution of this latter equation is

$$
G(x)=a e^{A x}+b e^{-A x}+d .
$$

As we have obtained a necessary condition for a function to satisfy (4.1) we may now substitute (4.17) into either the original equation or (4.7), placing restrictions on the constants. This leads to the solutions above.

For example, we may express the functions vanishing at $x=0$ by

$$
f(x)=\frac{(1-u(x))(u(x)-\beta)}{u(x)(1+\beta) c_{-}}
$$

where $u(x)=e^{x}$ and $c_{+} / c_{-}=-(1+\beta)^{2} /\left(1+\beta^{2}\right)$. Then from (4.12)

$$
f(4 x)=\frac{\left(1-u(x)^{4}\right)\left(u(x)^{4}-\beta^{4}\right)}{u(x)^{4}\left(1+\beta^{2}\right)(1+\beta)^{2} c_{-}}=\frac{(1-u(4 x))(u(4 x)-\beta)}{u(4 x)(1+\beta) c_{-}}
$$

Solving this leads to $\beta=0$ and the solution (4.5), or to $\beta=\omega, \omega^{2}$, where $\omega$ is a nontrivial cube-root of unity and the solution (4.4). (Here we have used that $u(x)=e^{x}$. However, when $\beta=0, \omega$, or $\omega^{2}$ and $u(0)=1$ we may in fact deduce that $u(x)=e^{x}$.)

\section{References}

[1] J. Aczél, Lectures on functional equations and their applications, Academic Press, New York 1966.

[2] R. Beals, D. Sattinger, J. Szmigielski, Multipeakons and the classical moment problem, Adv. Math. 154 (2000) 229-257.

[3] R. Beals, D. Sattinger, and J. Szmigielski, Multi-peakons and a theorem of Stieltjes, Inverse Problems 15 (1999) L1-L4.

[4] R. Beals, D. Sattinger, and J. Szmigielski, Peakon-antipeakon interaction, J. Nonlinear Math. Phys. 8 (2001) Suppl. 23-27. 
[5] H.W. Braden and J.G.B. Byatt-Smith, On a Functional Differential Equation of Determinantal Type, Bull. Lond. Math. Soc. 31 (1999) 463-470.

[6] R. Camassa and D.D. Holm, An integrable shallow water equation with peaked solitons, Phys. Rev. Lett. 71 (1993) 1661-1664.

[7] R. Camassa, D.D. Holm and J.M. Hyman, A New Integrable Shallow Water Equation, Advances in Applied Mechanics 31 (1994) 1-33.

[8] F. Calogero, Classical many-body problems amenable to exact treatments, Lecture Notes in Physics Monograph 66, Springer-Verlag (2001).

[9] F. Calogero and J.-P. Francoise, A completely integrable Hamiltonian system, J. Math. Phys. 37 (1996) 2863-2871.

[10] A. Constantin and H. P. McKean, A shallow water equation on the circle, Comm. Pure Appl. Math. 52 (1999) 949-982.

[11] A. Constantin and L. Molinet, Global weak solutions for a shallow water equation, Comm. Math. Phys. 211 (2000) 45-61.

[12] A. Constantin, On the scattering problem for the Camassa-Holm equation, R. Soc. Lond. Proc. Ser. A Math. Phys. Eng. Sci. 457 (2001) 953-970.

[13] A. Constantin and W. Strauss, Stability of peakons, Comm. Pure Appl. Math. 53 (2000) 603610.

[14] A. Constantin and L. Molinet, Orbital stability of solitary waves for a shallow water equation, Physica D 157 (2001) 75-89.

[15] A. Constantin and B. Kolev, On the geometric approach to the motion of inertial mechanical systems, J. Phys. A 35 (2002) R51-R79.

[16] A. Constantin and B. Kolev, Geodesic flow on the diffeomorphism group of the circle, Comment. Math. Helv. 78 (2003) 787-804.

[17] A. Degasperis and M. Procesi, Asymptotic integrability, in Symmetry and Perturbation Theory (eds. A. Degasperis and G. Gaeta), World Scientific (1999) 23-37.

[18] A. Degasperis, A.N.W. Hone and D.D. Holm, A New Integrable Equation with Peakon Solutions, Theoret. Math. Phys. 133 (2002) 1461-1472.

[19] A. Degasperis, A.N.W. Hone and D.D. Holm, Integrable and non-integrable equations with peakons, in Nonlinear Physics: Theory and Experiment II, eds. M.J. Ablowitz, M. Boiti, F. Pempinelli \& B. Prinari, World Scientific (2003) 37-43; nlin.SI/0209008

[20] H.R. Dullin, G.A. Gottwald and D.D. Holm, Camassa-Holm, Korteweg-de Vries-5 and other asymptotically equivalent equations for shallow water, Fluid Dynamics Research 33 (2003) 73-95.

[21] H.R. Dullin, G.A. Gottwald and D.D. Holm, On asymptotically equivalent shallow water wave equations, Physica D 190 (2004) 1-14.

[22] O. Fringer and D.D. Holm, Integrable vs. nonintegrable geodesic soliton behaviour, Physica D 150 (2001) 237-263.

[23] B. Fuchssteiner and A.S. Fokas, Symplectic structures, their Bäcklund transformations, and hereditary symmetries, Physica D 4 (1981) 47. 
[24] D.D. Holm and M.F. Staley, Wave Structures and Nonlinear Balances in a Family of $1+1$ Evolutionary PDEs, SIAM J. Appl. Dyn. Syst. 2 (2003) 323-380.

[25] D.D. Holm and A.N.W. Hone, Nonintegrability of a fifth order equation with integrable twobody dynamics, Theoret. Math. Phys. 137 (2003) 1459-1471.

[26] D.D. Holm and J.E. Marsden, Momentum Maps and Measure-valued Solutions (Peakons, Filaments and Sheets) for the EPDiff Equation, nlin.CD/0312048

[27] A.N.W. Hone, The associated Camassa-Holm equation and the KdV equation, J. Phys. A 32 (1999) L307-L314.

[28] A.N.W. Hone, Reciprocal link for 2+1-dimensional Extensions of Shallow Water Equations, Applied Mathematics Letters 13 (2000) 37-42.

[29] A.N.W. Hone and Jing Ping Wang, Prolongation algebras and Hamiltonian operators for peakon equations, Inverse Problems 19 (2003) 129-145.

[30] A.N.W. Hone, Painlevé tests, singularity structure and integrability, in What is Integrability?, ed. A.V. Mikhailov, Princeton University Press, to appear; preprint (2003) UKC/IMS/03/33.

[31] R. S. Johnson, Camassa-Holm, Korteweg-de Vries and related models for water waves, J. Fluid Mech. 455 (2002) 63-82.

[32] R.A. Kraenkel and A. Zenchuk, Two-dimensional integrable generalization of the CamassaHolm equation Phys. Lett. A 260 (1999) 218-224.

[33] A.V. Mikhailov and V.S. Novikov, Perturbative symmetry approach, J. Phys. A 35 (2002) 4775-4790.

[34] G. Misiolek, A shallow water equation as a geodesic flow on the Bott-Virasoro group, J. Geom. Phys. 24 (1998) 203-208.

[35] P.J. Olver, Applications of Lie Groups to Differential Equations, 2nd edition, Springer-Verlag (1993).

[36] P.J. Olver and P. Rosenau, Tri-Hamiltonian Duality Between Solitons and Compactons, Phys. Rev. E 53 (1996) 1900-1906.

[37] E.J. Parkes and V.O. Vakhnenko, The calculation of multi-soltion solutions of the Vakhnenko equation by the inverse scattering method, Chaos, Solitons and Fractals 13 (2002) 1819-1826.

[38] V.O. Vakhnenko, Solitons in a nonlinear model medium, J. Phys. A 25 (1992) 4181-4187. 